\title{
Relativistic Nonextensive Thermodynamics
}

\author{
A. Lavagno \\ Dipartimento di Fisica, Politecnico di Torino and INFN, Sezione di Torino \\ C.so Duca degli Abruzzi 24, I-10129 Torino, Italy
}

\begin{abstract}
Starting from the basic prescriptions of the Tsallis' nonextensive thermostatistics, i.e. generalized entropy and normalized $q$-expectation values, we study the relativistic nonextensive thermodynamics and derive a Boltzmann transport equation that implies the validity of the $H$-theorem where a local nonextensive four-entropy density is considered. Macroscopic thermodynamic functions and the equation of state for a perfect gas are derived at the equilibrium.
\end{abstract}

PACS: 05.20.Dd, 05.70.Ln, 05.90.+m, 25.75.-q

\section{Introduction}

Recently, there is an increasing evidence that the generalized nonextensive statistical mechanics, proposed by Tsallis [1], can be considered as the more appropriate basis of a theoretical framework to deal with physical phenomena where long-range interactions, long-range microscopic memories and/or fractal space-time constraints are present (cf. [1] for details). A considerable variety of physical applications involve a quantitative agreement between experimental data and theoretical models based on Tsallis' thermostatistics [2]. In particular there is a growing interest to high energy physics applications of nonextensive statistics. Several authors outline the possibility that experimental observations in relativistic heavy-ion collisions can reflect nonextensive features during the early stage of the collisions and the thermalization evolution of the system [3-7].

The basic aim of this letter is to study the nonextensive statistical mechanics formalism in the relativistic regime and to investigate, through an appropriate relativistic Boltzmann equation, the non-equilibrium and the equilibrium thermodynamics relations. 


\section{Basic assumptions in nonextensive thermostatistics}

Let us briefly review some basic assumptions of the nonextensive thermostatistics that will be useful in view of the relativistic extension.

Starting point of the Tsallis' generalization of the Boltzmann-Gibbs statistical mechanics is the introduction of a $q$-deformed entropy functional defined, in a phase space system, as [1]

$$
S_{q}=-k_{B} \int d \Omega p^{q} \ln _{q} p
$$

where $k_{B}$ is the Boltzmann constant, $p=p(x, v)$ is the phase space probability distribution, $d \Omega$ stands for the corresponding phase space volume element and $\ln _{q} x=\left(x^{1-q}-1\right) /(1-q)$ is, for $x>0$, the $q$-deformed logarithmic function. For the real parameter $q \rightarrow 1$, Eq.(1) reduces to the standard Boltzmann-Gibbs entropy functional.

In the equilibrium canonical ensemble, under the constraints imposed by the probability normalization

$$
\int d \Omega p=1
$$

and the normalized $q$-mean expectation value of the energy [8]

$$
\langle E\rangle_{q}=\frac{\int d \Omega p^{q} H(x, v)}{\int d \Omega p^{q}},
$$

the maximum entropy principle gives the probability distribution $[8,9]$

$$
p(x, v)=\frac{f(x, v)}{Z_{q}},
$$

where

$$
f(x, v)=\left[1-(1-q) \beta\left(H(x, v)-\langle H\rangle_{q}\right)\right]^{1 /(1-q)}
$$

and

$$
Z_{q}=\int d \Omega f(x, v)
$$

Let us note that, depending from the extremization procedure, in Ref.[8] the above factor $\beta$ is only proportional to the Lagrange multiplier, because of the 
probability distribution is self-referential, while, in Ref.[9], $\beta$ is actually the Lagrange multiplier associated to the energy constraint.

By using the definitions in Eqs. (4) and (6) into the relation $Z_{q}^{1-q}=\int d \Omega p^{q}$ [9], the following identity holds [10]

$$
\int d \Omega f(x, v) \equiv \int d \Omega f^{q}(x, v)
$$

and the normalized $q$-mean expectation value for a physical observable $A(x, v)$ can be expressed as

$$
\langle A\rangle_{q}=\frac{\int d \Omega f^{q} A(x, v)}{\int d \Omega f^{q}} \equiv \frac{1}{Z_{q}} \int d \Omega f^{q} A(x, v) .
$$

Therefore, the probability distribution and the $q$-mean value of an observable have the same normalization factor $Z_{q}$, as in the extensive statistical mechanics. Such a non-trivial property does not depend on the equilibrium frame but it comes from the normalization condition and holds at any time (if we require that the transport equation conserves the probability normalization or the number of particles). This observation will play a crucial role in the correct formulation of the relativistic Boltzmann equation and in the definition of the thermodynamic variables.

\section{Relativistic kinetic theory}

On the basis of the above prescriptions, we are able to progress in the formulation of the relativistic nonextensive statistical mechanics. Let us start defining the basic macroscopic variables in the language of relativistic kinetic theory. Because we are going to describe a non-uniform system in the phase space, we introduce the particle four-flow as

$$
N^{\mu}(x)=\frac{1}{Z_{q}} \int \frac{d^{3} p}{p^{0}} p^{\mu} f(x, p),
$$

and the energy-momentum flow as

$$
T^{\mu \nu}(x)=\frac{1}{Z_{q}} \int \frac{d^{3} p}{p^{0}} p^{\mu} p^{\nu} f^{q}(x, p),
$$

where we have set $\hbar=c=1, x \equiv x^{\mu}=(t, \mathbf{x}), p \equiv p^{\mu}=\left(p^{0}, \mathbf{p}\right)$ and $p^{0}=\sqrt{\mathbf{p}^{2}+m^{2}}$ is the relativistic energy. The four-vector $N^{\mu}=(n, \mathbf{j})$ contains the probability density $n=n(x)$ (which is normalized to unity) and 
the probability flow $\mathbf{j}=\mathbf{j}(x)$. The energy-momentum tensor contains the normalized $q$-mean expectation value of the energy density, as well as the energy flow, the momentum and the momentum flow per particle. Its expression follows directly from the definition (8); for this reason it is given in terms of $f^{q}(x, p)$.

In order to derive a relativistic Boltzmann equation for a dilute system in nonextensive statistical mechanics, we consider the finite volume elements $\Delta^{3} x$ and $\Delta^{3} p$ in the phase space. These volume elements are large enough to contain a very large number of particles but also small enough compared to the macroscopic dimension of the system. If, in the volume $\Delta^{3} x \Delta^{3} p$, is contained a representative sample of system, we can assume that Eq.(7) still holds in such phase space portion. Then, in the Lorentz frame, the particle fraction $\Delta N(x, p)$ in the volume $\Delta^{3} x \Delta^{3} p$ can be written as

$$
\Delta N(x, p)=\frac{N}{Z_{q}} \int_{\Delta^{3} x} \int_{\Delta^{3} p} d \Omega f^{q}(x, p),
$$

where $N$ is the total number of particles of the system. With respect to the observer's frame of reference, the above expression becomes

$$
\Delta N(x, p)=\frac{N}{Z_{q}} \int_{\Delta^{3} \sigma \Delta^{3} p} \int_{d^{3}} \sigma_{\mu} \frac{d^{3} p}{p^{0}} p^{\mu} f^{q}(x, p),
$$

where $d^{3} \sigma_{\mu}$ is a time-like three-surface element of a plane space-like surface $\sigma$ [11]. Requiring that the net flow through the surface $\Delta^{3} \sigma$ of the element $\Delta^{4} x$ vanishes in absence of collisions, we have: $p^{\mu} \partial_{\mu} f^{q}(x, p)=0$. While considering collisions between particles, the Boltzmann equation becomes

$$
p^{\mu} \partial_{\mu} f^{q}(x, p)=C_{q}(x, p),
$$

where $C_{q}(x, p)$ is the $q$-deformed collision term that, under the hypothesis that only binary collisions occur in the gas, can be expressed as

$$
\begin{aligned}
C_{q}(x, p)= & \frac{1}{2} \int \frac{d^{3} p_{1}}{p_{1}^{0}} \frac{d^{3} p^{\prime}}{p^{\prime 0}} \frac{d^{3} p_{1}^{\prime}}{p_{1}^{\prime 0}}\left\{h_{q}\left[f^{\prime}, f_{1}^{\prime}\right] W\left(p^{\prime}, p_{1}^{\prime} \mid p, p_{1}\right)\right. \\
& \left.-h_{q}\left[f, f_{1}\right] W\left(p, p_{1} \mid p^{\prime}, p^{\prime}{ }_{1}\right)\right\} .
\end{aligned}
$$

In the above equation, we have set $W\left(p, p_{1} \mid p^{\prime}, p^{\prime}{ }_{1}\right)$ as the transition rate between two particle state with initial four-momentum $p$ and $p^{\prime}$ and a final state with four-momenta $p_{1}$ and $p_{1}^{\prime} ; h_{q}\left[f, f_{1}\right]$ is the correlation function related to two particles in the same space-time position but with different four-momenta 
$p$ and $p_{1}$, respectively. The factorization of $h_{q}$ in two single probability distributions (uncorrelated particles at the same spatial point) is the celebrated hypothesis of molecular chaos (Boltzmann's Stosszahlansatz). Thus, the function $h_{q}$ defines implicitly a generalized nonextensive molecular chaos hypothesis.

By assuming the conservation of the energy-momentum in the collisions (i.e. $\left.p^{\mu}+p^{\prime \mu}=p_{1}^{\mu}+p_{1}^{\prime \mu}\right)$ and requiring that the correlation function $h_{q}$ is symmetric and always positive $\left(h_{q}\left[f, f_{1}\right]=h_{q}\left[f_{1}, f\right], h_{q}\left[f, f_{1}\right]>0\right)$, it is easy to show that collision term satisfies the following property

$$
F[\psi]=\int \frac{d^{3} p}{p^{0}} \psi(x, p) C_{q}(x, p)=0
$$

if

$$
\psi(x, p)=a(x)+b_{\mu}(x) p^{\mu}
$$

where $a(x)$ and $b(x)$ are arbitrary functions.

By choosing $\psi=$ const. and using the Boltzmann equation (13), then Eq.(15) implies

$$
\frac{\partial}{\partial t} \int d \Omega f^{q}(x, p)=0
$$

and this is, on account of Eq.(7), nothing else that the conservation of the probability normalization $Z_{q}$. Otherwise, by setting $\psi=b_{\mu} p^{\mu}$, we have from Eq.(15)

$$
\partial_{\nu} T^{\mu \nu}(x)=0
$$

which implies the energy and the momentum conservation.

Let us remark that to have conservation of the probability normalization, energy and momentum, it is crucial that not only the collision term $C_{q}$ be explicitly deformed by means of the function $h_{q}$, but also the streaming term $p^{\mu} \partial_{\mu} f^{q}$. This matter of fact is a directly consequence of the nonextensive statistical prescription of the normalized $q$-mean expectation value (8) and is not taken into account in the non-relativistic formulation of Ref.[12]. 


\section{Local $H$-theorem}

The relativistic local $H$-theorem states that the entropy production $\sigma_{q}(x)=$ $\partial_{\mu} S_{q}^{\mu}(x)$ at any space-time point is never negative.

Assuming the validity of the Tsallis entropy (1), it appears natural to introduce the nonextensive four-flow entropy $S_{q}^{\mu}(x)$ as follows

$$
S_{q}^{\mu}(x)=-k_{B} \int \frac{d^{3} p}{p^{0}} p^{\mu} f^{q}(x, p)\left[\ln _{q} f(x, p)-1\right] .
$$

On the basis of the above equation the entropy production can be written as

$$
\sigma_{q}(x)=-k_{B} \int \frac{d^{3} p}{p^{0}} \ln _{q} f p^{\mu} \partial_{\mu} f^{q} \equiv-k_{B} F\left[\ln _{q} f\right],
$$

where the second identity follows from the Boltzmann equation (13) and the definition of $F[\psi]$ in Eq.(15). After simple manipulations, Eq.(20) can be rewritten as

$$
\begin{aligned}
\sigma_{q}(x)= & \frac{k_{B}}{8} \int \frac{d^{3} p_{1}}{p_{1}^{0}} \frac{d^{3} p_{1}}{p_{1}^{0}} \frac{d^{3} p^{\prime}}{p^{\prime}} \frac{d^{3} p_{1}{ }^{\prime}}{p_{1}^{\prime 0}}\left(\ln _{q} f^{\prime}+\ln _{q} f^{\prime}{ }_{1}-\ln _{q} f+\ln _{q} f_{1}\right) \times \\
& \left\{h_{q}\left[f^{\prime}, f^{\prime}{ }_{1}\right] W\left(p^{\prime}, p^{\prime}{ }_{1} \mid p, p_{1}\right)-h_{q}\left[f, f_{1}\right] W\left(p, p_{1} \mid p^{\prime}, p^{\prime}{ }_{1}\right)\right\} .
\end{aligned}
$$

By assuming the detailed-balance property $W\left(p, p_{1} \mid p^{\prime}, p_{1}^{\prime}\right)=W\left(p^{\prime}, p_{1}^{\prime} \mid p, p_{1}\right)$, we have that the entropy production is always an increasing function, if $q>0$ and if the function $h_{q}$ satisfies the general condition

$$
h_{q}\left[f, f_{1}\right]=h_{q}\left[\ln _{q} f+\ln _{q} f_{1}\right],
$$

in addition to be symmetric and always positive.

Because the nonextensive formalism reduces to the standard Boltzmann kinetic formulation for $q \rightarrow 1$, it appears natural to postulate the $q$-generalized Boltzmann molecular chaos hypothesis as

$$
h_{q}\left[f, f_{1}\right]=e_{q}\left(\ln _{q} f+\ln _{q} f_{1}\right),
$$

where we have introduced the Tsallis $q$-exponential function

$$
e_{q}(x)=[1+(1-q) x]^{1 /(1-q)},
$$


which satisfies the properties: $e_{q}\left(\ln _{q} x\right)=x$ and $e_{q}(x) \cdot e_{q}(y)=e_{q}[x+y+$ $(1-q) x y$. Let us note that a similar expression for the function $h_{q}$ was previously introduced in Ref.s[12,13] and a rigorous justification of the validity of the ansatz (23) can be found only by means of a microscopic analysis of the dynamics of correlations in nonextensive statistics.

\section{Equilibrium and equation of state}

The condition that entropy production vanishes everywhere, together with requirement that the equilibrium probability distribution $f_{e q}$ must be a solution of the transport equation (13), uniquely defines the state of equilibrium. Taking into account of Eqs.(15), (16) and (20), the condition $\sigma_{q}=0$ can only occur when $\ln _{q} f_{e q}=a+b_{\mu} p^{\mu}$. By imposing that $f_{e q}$ must satisfy Eq.(13) and after simple redefinition of the coefficients $a$ and $b$, the equilibrium probability distribution can be written as a Tsallis-like distribution

$$
f_{e q}(p)=\frac{1}{Z_{q}}\left[1-(1-q) \frac{p^{\mu} U_{\mu}}{k_{B} T}\right]^{1 /(1-q)},
$$

where $U_{\mu}$ is the hydrodynamic four-velocity [11] and $f_{e q}$ depends only on the momentum in absence of an external field. At this stage, $k_{B} T$ is a free parameter and only in the derivation of the equation of state it will be identified with the physical temperature. Moreover, it is easy to show that $f_{e q}$ is a solution of the transport equation (13).

We are able now to evaluate explicitly all other thermodynamic variables and provide a complete macroscopic description of a relativistic system at the equilibrium. Let us first calculate the probability density defined as

$$
n=N^{\mu} U_{\mu}=\frac{1}{Z_{q}} \int \frac{d^{3} p}{p^{0}} p^{\mu} U_{\mu} f_{e q}(p) .
$$

Since $n$ is a scalar, it can be evaluated in the rest frame where $U^{\mu}=(1,0,0,0)$. Setting $\tau=p^{0} / k_{B} T$ and $z=m / k_{B} T$, the above integral can be written as

$$
\begin{aligned}
n & =\frac{4 \pi}{Z_{q}}\left(k_{B} T\right)^{3} \int_{z}^{\infty} d \tau\left(\tau^{2}-z^{2}\right)^{1 / 2} \tau e_{q}^{-\tau} \\
& =\frac{4 \pi}{3 Z_{q}}\left(k_{B} T\right)^{3} \int_{z}^{\infty} d \tau\left(\tau^{2}-z^{2}\right)^{3 / 2}\left(e_{q}^{-\tau}\right)^{q}
\end{aligned}
$$


where the last identity has been obtained by a partial integration. Let us introduce the $q$-modified Bessel function of the second kind as follows

$$
K_{n}(q, z)=\frac{2^{n} n !}{(2 n) !} \frac{1}{z^{n}} \int_{z}^{\infty} d \tau\left(\tau^{2}-z^{2}\right)^{n-1 / 2}\left(e_{q}^{-\tau}\right)^{q}
$$

then, the particle density can be cast into the compact form

$$
n=\frac{4 \pi}{Z_{q}} m^{2} k_{B} T K_{2}(q, z) .
$$

Similarly we can obtain the other macroscopic thermodynamic variables. Considering the decomposition of the energy-momentum tensor [11]: $T^{\mu \nu}=\epsilon U^{\mu} U^{\nu}$ $p \Delta^{\mu \nu}$, where $\epsilon$ is the energy density, $p$ the pressure and $\Delta^{\mu \nu}=g^{\mu \nu}-U^{\mu} U^{\nu}$, the equilibrium pressure can be calculated as

$$
p=-\frac{1}{3} T^{\mu \nu} \Delta_{\mu \nu}=-\frac{1}{3 Z_{q}} \int \frac{d^{3} p}{p^{0}} p^{\mu} p^{\nu} \Delta_{\mu \nu} f_{e q}^{q}(p),
$$

and can be expressed as

$$
p=\frac{4 \pi}{Z_{q}} m^{2}\left(k_{B} T\right)^{2} K_{2}(q, z) .
$$

Comparing Eq.(29) with Eq.(31), we obtain

$$
p=n k_{B} T,
$$

which is the equation of state of a perfect gas if we identify $T$ as the physical temperature of the system. An explicit $q$-dependence does not appear. The same equation has been derived in non-relativistic regime and in nonextensive scenario $[10,14]$.

We proceed now to calculate the energy density $\epsilon$ as

$$
\epsilon=T^{\mu \nu} U_{\mu} U_{\nu}=\frac{1}{Z_{q}} \int \frac{d^{3} p}{p^{0}}\left(p^{\mu} U_{\mu}\right)^{2} f_{e q}^{q}(p)
$$

Inserting the previously defined variables $\tau$ and $z$ and using the definition in Eq.(28), we obtain

$$
\epsilon=\frac{4 \pi}{Z_{q}} m^{4}\left[3 \frac{K_{2}(q, z)}{z^{2}}+\frac{K_{1}(q, z)}{z}\right] .
$$


Thus the energy per particle $e=\epsilon / n$ is

$$
e=3 k_{B} T+m \frac{K_{1}(q, z)}{K_{2}(q, z)}
$$

which has the same structure of the relativistic expression obtained in the framework of the equilibrium Boltzmann-Gibbs statistics [11].

In the non-relativistic limit $(p \ll 1)$ the energy per particle reduces to the well-known expression

$$
e \simeq m+\frac{3}{2} k_{B} T
$$

Also in this case, no explicit $q$-dependence is detected.

\section{Conclusion}

The physical motivation of this investigation lies in the strong relevance that nonextensive statistics could have in high energy physics. In this letter we have studied the thermostatistics of a relativistic system in nonextensive statistics; the obtained results can be easily extended to the case where an external force is present.

The prescription of the normalized $q$-mean expectation values implies a consistent nonextensive generalization of the macroscopic variables $N^{\mu}$ and $T^{\mu \nu}$. On this basis, we have derived a generalized Boltzmann equation where both the streaming and the collision terms depend on the deformation parameter $q$. Such a transport equation conserves the probability normalization (or number of particles) and is consistent with the energy-momentum conservation laws. The collision term contains a generalized expression of the molecular chaos and for $q>0$ implies the validity of a generalized $H$-theorem, if the nonextensive local four-density entropy (19) is assumed. At the equilibrium, the solution of the Boltzmann equation is a relativistic Tsallis-like distribution and the equation of state of a classical relativistic gas in nonextensive statistical mechanics has the same form as in ordinary Boltzmann-Gibbs frame.

Finally, nonextensive statistical effects can be detected in connection to microscopic observables such as particle distribution, correlation functions, fluctuations of thermodynamical variables but not directly in connection to macroscopic variables, such as temperature or pressure, because the equation of state and the macroscopic thermodynamical relations do not explicitly depend on 
the deformation parameter $q$.

\section{Acknowledgements}

It is a pleasure to thank P. Quarati and C. Tsallis for useful discussions.

\section{References}

[1] C. Tsallis, J. Stat. Phys. 52, 479 (1988). See also: Nonextensive Statistical Mechanics and Its Applications, Editors S. Abe, Y. Okamoto, Springer Verlag, 2001.

[2] See http://tsallis.cat.cbpf.br/biblio.htm for a regularly updated bibliography on the subject.

[3] W.M. Alberico, A. Lavagno, P. Quarati, Eur. Phys. J. C 12 (2000) 499; W.M. Alberico, A. Lavagno, P. Quarati, Nucl. Phys. A 680 (2001) 94; A. Lavagno, P. Quarati, Chaos, Solitons and Fractals 13 (2002) 569; A. Lavagno, Physica A 305 (2002) 238.

[4] G. Wilk, Z. Wlodarczyk, Phys. Rev. Lett. 84 (2000) 2770; O.V. Utyuzh, G. Wilk, Z. Wlodarczyk, J. Phys. G 26 (2000) L39.

[5] D.B. Walton, J. Rafelski, Phys. Rev. Lett. 84 (2000) 31.

[6] I. Bediaga, E.M.F. Curado, J.M. de Miranda, Physica A 286 (2000) 156.

[7] C. Beck, Physica A 286 (2000) 164.

[8] C. Tsallis, R.S. Mendes, A.R. Plastino, Physica A 261 (1998) 534.

[9] S. Martínez, F. Nicolás, F. Pennini, A. Plastino, Physica A 286 (2000) 489.

[10] S. Martínez, F. Pennini, A. Plastino, Phys. Lett. A 278 (2000) 47.

[11] S.R. Groot, W.A. van Leeuwen, Ch. G. van Weert, Relativistic kinetic theory, North-Holland, 1980.

[12] J.A.S. Lima, R. Silva, A.R. Plastino, Phys. Rev. Lett. 86 (2001) 2938.

[13] G. Kaniadakis, Physica A 296 (2001) 405; Phys. Lett. A 288 (2001) 283.

[14] S. Abe, S. Martínez, F. Pennini, A. Plastino, Phys. Lett. A 281 (2001) 126. 